Afrika Focus, Vol. 9, Nr. 1-2, 1993, pp.16-41

\title{
KAZANGA - ETHNICITÉ EN AFRIQUE ENTRE ÉTAT ET TRADITION
}

\section{Wim VAN BINSBERGEN}

Centre d'Etudes Africaine, B.P. 9555, 2300 RB Leyde, Pays-Bas /

Département d'Anthropologie et de Sociologie de Développement, Université libre d'Amsterdam

\section{SUMMARY}

\section{KAZANGA - ETHNICITY IN AFRICA BETWEEN STATE AND TRADITION}

The production of cultural forms at the interface between a rural-based tradition and the state is a familiar aspect of ethnicity in contemporary Africa. This paper seeks to identify some of the characteristics of this process, whose products are too often misunderstood, and cherished, as 'authentic' forms of 'tradition'. Highlighting the role of ethnic brokers, of the modern mass media, and of a model of commoditified 'performance' as an aspect of contemporary electronic mass culture, the argument explores the production of expressive culture in the context of the Kazanga cultural association and its Kazanga annual festival among the Nkoya people of central western Zambia since the early 1980s, against the background of Nkoya ethnicity and Nkoya expressive and court culture since the 19 th century.

KEY WORDS: associations, brokers, commoditification, dance, ethnicity, festivals, music, Nkoya, state, Zambia 


\section{Ethnicité (1)}

Un aspect très ancien et probablement universel de la condition humaine est le fait que nous donnons des noms (2) non seulement à des formes non humaines et à des individus humains, mais aussi aux relations suivant lesquelles nous nous organisons (3). Les membres d'une communauté locale définissent généralement la relation sociale propre à l'aide d'un nom propre et, en tous cas, attribuent des noms à d'autres communautés. Bien que souvent vague, une telle nomenclature crée une ordonnance dramatique à l'intérieur du champ social que se partagent plusieurs communautés. L'attribution d'un nom particulier qui ne s'applique pas au propre dénie à l'autre la possibilité de différer du propre seulement d'une façon graduelle. L'opposition est absolutisée verbalement par le nom et est soumise en principe à la rigueur du dendrogramme et des oppositions binaires qui jouent un rôle si important dans la pensée humaine (4). En nommant l'autre catégorie ' $A$ ', l'ensemble propre s'identifie en tous cas comme 'non $\mathbf{A}$ '.

Chaque société comprend de nombreux ensembles humains portant un nom, comme par exemple les communautés locales, les groupes de parenté, les groupes de production, les éléments d'un appareil administratif, les cultes et les organisations bénévoles. Nous ne voudrions appeler ces ensembles 'groupes ethniques' qu'en présence de certaines caractéristiques supplémentaires: premièrement, si l'appartenance individuelle est primairement empruntée au droit de naissance (ascription); deuxièmement, si l'ensemble humain se distingue consciemment et explicitement d'autres ensembles voisins similaires par des différences culturelles spécifiques; et finalement, si les membres s'identifient les uns aux autres sur la base d'une conscience d'une expérience historique commune. L'ethnicité' est alors la totalité des processus par lesquels les hommes structurent le large champ social et géographique dans lequel ils sont impliqués en un champ ethnique, en référence aux groupes ethniques qu'ils distinguent.

La nomenclature ethnique est un processus social complexe qui justifie à une recherche spécifique. Cette idée n'est pas en vogue depuis très longtemps en anthropologie. Jusqu'au milieu du vingtième siècle, les chercheurs utilisaient des noms ethniques comme étiquettes pour marquer des unités de culture et d'organisation apparemment évidentes: des recherches ont été faites à l'intérieur, mais la délimitation elle-même a été à peine problématisée. Ce n'est que dans les années 1960 que la notion de 'tribu', comme expression ethnocentrique et réifiée d'un groupe ethnique à l'intérieur d'un champ ethnique mondial mais à l'extérieur de la civilisation politiquement dominante, a été soumise à une critique approfondie. 
On a beaucoup écrit depuis sur le développement et le déclin de la notion de tribu en Afrique dans le cadre des processus politiques et économiques qui se sont déroulés sur ce continent depuis la fin du dix-neuvième siècle (5). Mais nous en savons encore très peu pour l'instant sur les processus de transformation symbolique et culturelle dont a usé l'ethnicité dans ce contexte. C'est la question que je voudrais soulever ici.

\section{Identité ethnique et médiation ethnique}

Le terme d' 'identité' est très souvent utilisé dans le cadre de l'ethnicité et des recherches sur l'ethnicité (6). L'identité' peut se définir comme étant la perception socialement construite du moi en tant que membre du groupe. Chaque personne joue plusieurs rôles dans divers groupes et a, de ce fait, une multiplicité d'identités formées au cours de sa socialisation comme membre de ces groupes.

Le développement d'un groupe ethnique en Afrique consiste, généralement, en un projet qui promeut une nouvelle identité et la met en place dans la personnalité des membres visés. Le projet d'ethnisation présente l'identité ethnique (exprimée par le nom de groupe) comme une identité ultime, dominante et ancrée au plus profond, devant comprendre toutes les autres identités que l'on a acquises en tant que membre de la société locale.

L'ethnicité comprend notamment un processus de prise de conscience dans le cadre duquel des identités diffuses sont regroupées sous le dénominateur d'une seule identité ethnique, marquée d'un nom spécifique. Le nom est construit comme limite culturelle, et la culture est reconstruite pour s'inscrire à l'intérieur de cette limite et fournir des attributs culturels distinctifs. La conjugaison et le réarrangement des identités transforment l'expérience personnelle du moi et du monde: la découverte de 'je suis - flamand, azeri, yoruba, nkoya, etc.' offre une perspective de classification dans laquelle l'impuissance, l'aliénation et les privations vécues dans de nombreuses situations apparaissent sous un jour nouveau - l'espoir que cette autoprésentation ethnique transforme ces expériences négatives en leur contraire. Ainsi, l'ethnicité présente de nombreux parallèles avec d'autres phénomènes idéologiques comme le nationalisme, le réveil de la conscience de classe, la conversion et le renouveau religieux.

L'éthnicité présente une dialectique remarquable que je suis enclin à considérer comme son moteur (7). La structure logique de la désignation est encore durcie par ascription et se prétend inconditionnelle, délimitée, inévitable et intemporelle (8); 
en revanche, la construction d'une culture coextensive à la limite du groupe, de symboles culturels distinctifs et d'une conscience historique partagée, et le fait d'une appartenance partiellement non ascriptive - bref, la mise en place concrète de l'ethnicité dans le temps, signifient flexibilité, choix, faisabilité et changement récent. Ces deux aspects absolument contradictoires sont mis en question dans l'ethnicité. Cette dialectique rend l'ethnicité apte par excellence à jouer, dans les processus de changement, un rôle de médiateur entre des relations sociales structurées fondamentalement différentes, notamment entre le niveau local d'un côté, et l'Etat et les larges structures économiques de l'autre (9). Le nom ethnique et le principe d'ascription donnent l'image d'un ensemble humain délimité. Ainsi, l'intégration dans l'ensemble plus grand et autrement structuré n'est pas un problème que l'individu vulnérable et préparé à la communauté locale doit résoudre seul, mais devient l'objet d'une action de groupe. Un ensemble humain est structuré vers l'intérieur en groupe ethnique par la création d'un bagage culturel qui forme en soi un enjeu important dans la négociation entre le groupe ethnique en développement et le monde extérieur. On se distancie des groupes ethniques concurrents en mettant un accent stratégique sur des éléments culturels et linguistiques; et à un niveau plus large d'organisation socio-politique, on essaie d'obtenir de l'Etat des avantages politiques et économiques par la reconnaissance étatique du bagage ethnique construit.

Ce processus permet au groupe ethnique de s'articuler de plus en plus comme tel. Mais, bien que toutes les personnes impliquées dans ce processus soient égales en tant que détenteurs de l'identité ethnique, le contact avec le monde extérieur, en particulier dans le cas où il est couronné de succès, engendre de nouvelles inégalités à l'intérieur du groupe. La médiation se déroule par l'intermédiaire de médiateurs politiques, économiques et idéologiques qui (par plus de savoir, d'éducation, d'expérience, de contacts politiques et de moyens de les entretenir) savent mieux que les autres membres du groupe mettre à profit les possibilités offertes par le monde extérieur (10). Ces médiateurs développent la direction du groupe ethnique en un instrument de pouvoir qui va dans deux directions:

- vers le monde extérieur, où l'on se procure des ressources en échange de l'ordonnance effective du domaine local (11);

- et à l'intérieur du groupe ethnique, où les médiateurs éclusent de façon limitée les ressources acquises à l'extérieur en échange d'une autorité interne, d'un prestige et d'un contrôle de la production au niveau local.

Dans ce contexte de médiation entre la communauté locale et le monde extérieur, le propre en tant que propre devient problématique et l'assertion de la culture 'traditionnelle' et 'authentique' (mais en fait reconstruite) apparait comme une tâche importante et une source de pouvoir pour les médiateurs. Les associations, 
publications et manifestations ethniques organisées par les médiateurs, comme par exemple les festivals, constituent dans ce processus une formule largement répandue et éprouvée.

L'insistance sur l'identité ethnique produit des revendications idéologiques fortes pour lesquelles le monde extérieur manifeste parfois plus de sympathie que de compréhension analytique. On ne les reconnaît pas comme étant un produit stratégique et rhétorique récent, mais on les idéalise (comme le font les médiateurs ethniques eux-mêmes) comme étant l'expression courageuse d'une identité inéluctable, acquise par socialisation pendant l'enfance et qui résiste désespérément à l'assaut du monde extérieur. Dans la pensée actuelle sur l'aide au développement, on concède une place certaine à de telles expressions culturelles.

S'agit-il vraiment ici de la médiation d'une tradition profondément ancrée? Les processus ethniques ont-ils droit, de ce fait, à la sympathie et à l'appui que nous sommes enclins, dans un monde en plein changement, à donner à un bien culturel menacé? Comment ces expressions ethniques rendent-elles manifestes les détails du processus de négociation entre le monde extérieur et la communauté locale? Comment de nouvelles inégalités y trouvent-elles une expression? Y a t-il des arguments à trouver pour la thèse classique des chercheurs et hommes politiques marxistes selon laquelle le processus ethnique produit une fausse conscience qui masque aux participants l'exploitation sous-jacente, interprétable en termes de classe? (12) Que nous apprend l'analyse du processus de négociation ethnique sur le large système politique et économique actuel dans lequel il s'insère?

A présent, je vais porter témoignage d'une fête ethnique au coeur de l'Afrique, à laquelle ces questions s'appliquent de façon exemplaire.

\section{Le développement des Nkoya en groupe ethnique et l'association culturelle} 'Kazanga' (13)

Depuis 1988, une cérémonie remarquable, appelée Kazanga, a lieu chaque année le 1er juillet à Shikombwe, dans le district de Kaoma, dans l'Ouest de la république de Zambie.

Shikombwe est le village principal de Mwene Mutondo. Mwene signifie 'roi'. Shikombwe se reconnaît comme résidence royale à sa lilapa, palissade de roseaux soutenue par des poteaux pointus et réservés aux rois. L'intérieur se compose d'un très simple appartement de quatre pièces qui sert de palais royal, d'un hall 
d'audience en roseau et d'un auvent sous lequel les instruments de musique de l'orchestre royal - insignes royaux les plus en vue - sont mis à l'abri et joués deux fois par jour. Sur un grand espace libre situé hors de l'enceinte, se trouve le palais de justice moderne devant la porte duquel se dresse une hampe grossièrement taillée sur laquelle les agents de police désignés à la cour royale, les kapasus, hissent chaque jour le drapeau zambien. C'est ici que se déroule le festival. Les résidences des courtisans et des membres de la famille royale sont situées autour de ces deux places centrales. Un chemin de sable long de quinze kilomètres relie le village à la route bitumée qui mène au centre de district Kaoma, appelé Mankoya jusqu'en 1969, situé vingt kilomètres plus loin. Mongu et Lealui sont deux centres gouvernementaux, l'un moderne et l'autre traditionnel, de la Province occidentale (l'ancien Barotseland) dont fait partie le district de Kaoma et sont situés à deux cents kilomètres à l'Ouest; Lusaka, la capitale nationale de la Zambie, est à quatre cents kilomètres à l'Est. Le territoire de Mutondo, dont la superficie est égale à celle de quelques départements français, est couvert d'une savane fertile parsemée de hameaux habités par des petits paysans. De nombreux habitants se considèrent comme des sujets de Mutondo, comme Nkoya, et parlent de préférence la langue nkoya qui en générale n'est pas leur seule langue; d'autres se comptent parmi les Lozi(14), groupe dominant dans l'Ouest de la Zambie, ou parmi les groupes de migrants qui ont massivement quitté l'Angola depuis le début du vingtième siècle: surtout les Luvale (15) et les Luchazi.

Mutondo emprunte son statut royal à un royaume que ses ancêtres, dissidents de l'illustre royaume lunda dans le Sud du Zaïre, fondèrent dans cette région au dix-huitième siècle. A l'origine (16) nom d'un bois qui entourait le confluent des fleuves Zambèze et Kabompo, le nom nkoya est passé au groupe dynastique. A l'intérieur de la continuité culturelle de la région, 'nkoya' ne désignait pas une culture particulière et délimitée; les Nkoya n'étaient pas encore constitués en groupe ethnique. Devenus tributaires du roi de Barotseland au milieu du dix-neuvième siècle, l'Etat et les sujets de Mutondo ont été intégrés en 1900 en tant que 'tribu soumise aux Lozi', sous le nom de Mankoya, à l'Etat colonial qui deviendrait en 1964 la Zambie indépendante. Mutondo devint alors un titre éminent dans l'aristocratie lozi. Actuellement, la cour est toujours rétribuée par l'Etat national, dans le cadre des accords qu'il conclut en 1900 et 1964 avec le roi lozi.

Néanmoins, les relations Lozi-Nkoya sont vécues par ces derniers comme étant antagonistes et humiliantes, en particulier sous l'Etat colonial. Celui-ci laissa une grande liberté au gouvernement indigène lozi. Le district de Mankoya soupira sous la domination lozi. Hormis Mutondo, un seul autre souverain apparenté de la région, Mwene Kahare, survécut au processus d'incorporation dans l'Etat lozi. Les 
nombreux autres dignitaires furent remplacés par des gérants lozi. Deux rois intimement liés à la dynastie Mutondo, Mwene Kabulwebulwe et Mwene Momba, déplacèrent à temps leur siège hors du Barotseland; ils étaient reconnus depuis le début par l'Etat colonial mais ne partagaient pas, bien-sûr, la subvention accordée aux Lozi.

Les années décisives pour le développement des Nkoya en groupe ethnique conscient de soi ont été 1937, lorsque le roi lozi créa une dépendance de sa cour pour gérer au niveau du district l'autorité des chefs locaux, la justice et les finances,(17) et 1947, lorsque Mutondo Muchayila fut destitué par le roi lozi et exilé pour dix ans pour cause de rébellion. A la même époque, Johasaphat Shimunika, le premier pasteur autochtone de l'Eglise évangélique de Zambie (18), traduisit le Nouveau testament et les Psaumes (19) dans la langue locale qui serait appelée également 'nkoya'. Malgré les efforts de l'Eglise, cette langue, dont les usagers représentaient moins de $1 \%$ de la population zambienne, ne parvint pas à se faire reconnaître, on le comprend, dans l'enseignement et les médias: outre l'anglais qui est la langue officielle, la Zambie reconnaît déjà sept langues régionales parmi lesquelles le lozi. De plus, dans les années 1950-60, Shimunika mit par écrit les traditions orales, ce qui dota l'identité nkoya en développement (20) d'un passé glorieux. Dans sa tentative pan-nkoya, il impliqua expressément, aux côtés de Mutondo, les rois Kahare, Kabulwebulwe et Momba et leurs sujets, et déclara la domination lozi historiquement illégitime.

Les Nkoya en formation virent la lutte pour l'indépendance nationale comme une occasion de mettre fin à la domination régionale lozi. Ainsi, ils se tinrent à l'écart, les premières années, de l'Etat national dominé par le Parti pour l'Union nationale pour l'indépendance (UNIP), parti dominé localement par les Lozi. Grâce au recul des Lozi dans la politique nationale à partir de 1969 (21) et à la discorde qui régnait dans le district parmi les électeurs luvale et luchazi, les Nkoya obtinrent, aux élections générales de 1973, leur premier et unique siège au parlement et au ministère. Effrayé par le tribalisme, le gouvernement réagissait toujours de façon extrêmement négative aux expressions de l'ethnicité nkoya. A la même époque, un grand projet de développement fut entrepris dans l'Est du district. Les villageois en profitèrent à peine, contrairement à certains fermiers entreprenants qui affluèrent des autres districts et aux membres (étroitement apparentés) de l'élite politique moderne et traditionnelle des Nkoya. Cette élite éveilla l'enthousiasme et la loyalité des villageois grâce à la formulation de buts ethniques tels que l'augmentation des subventions aux rois reconnus, la réinstallation des chefferies disparues, et le plaidoyer pour la langue nkoya dans les médias et l'enseignement. Sous sa direction, le développement des départements locaux UNIP rendit 
l'expression de l'ethnicité nkoya acceptable pour l'Etat central. Pour la première fois, l'hymne national et les chants de lutte UNIP retentirent en langue nkoya.

Depuis le début du vingtième siècle, la vie des habitants de cette région ne se déroulait pas seulement dans les villages mais aussi dans les fermes, les mines et les régions urbaines de Zambie, du Zimbabwe et d'Afrique du Sud. Pendant longtemps, la composante urbaine de la communauté villageoise ne fut pas formalisée en association ethnique comme il en prospérait tant dans la Zambie coloniale. Ce n'est qu'en 1982 que 'l'association culturelle Kazanga' obtint la personalité juridique, sous le parrainage du ministre nkoya. L'initiative était due à une poignée de compatriotes d'âge moyen qui, malgré tous les handicaps, s'étaient transformés de migrants cycliques peu assurés en cadres moyens de la capitale. L'enthousiasme de plus en plus évident pour l'identité nkoya assurait à ces citadins des relations étroites avec l'élite politique du district et une nouvelle crédibilité aux yeux des villageois dont ils s'étaient distingués par leur position de classe et leur urbanisation. Ils reprirent les buts ethniques cités. Cependant, l'objectif principal de Kazanga était la propagation, grâce à un festival annuel du même nom, de la culture locale qui, bien-sûr, reçut l'étiquette 'nkoya'. Du nom d'un bois, le mot nkoya s'était étendu, par l'intermédiaire du nom dynastique et du nom de district, à un groupe ethnique représenté dans plusieurs districts, à une langue, à une culture et à un projet culturel qui devait faire reconnaître ce nouveau groupe au niveau régional et national.

\section{Le festival Kazanga de 1989}

Je vais me limiter maintenant à la description du festival Kazanga de 1989, sur lequel je dispose de données détaillées.

Sur le terrain libre près du palais de justice, sont installés pour les spectateurs des auvents de roseau et deux loges: l'une pour les rois et l'autre, voisine mais séparée par un paravent de roseau, pour une poignée de dignitaires d'Etat, parmi lesquels deux ministres (22). La stratégie bilatérale de la médiation ethnique ne pouvait pas mieux s'exprimer: la construction de l'identité ethnique vers la loge royale va de pair, le long d'un axe parallèle, avec l'assertion de cette identité vers la loge gouvernementale.

L'absence décevante des médias rend inutile l'installation de dispositifs d'enregistrement spéciaux. Néanmoins, les hauts-parleurs ne cessent de produire 
un effet Larsen et ne laissent aucun doute sur le fait que les produits locaux, musique, chant et danse, sont présentés dans une dimension inhabituelle. Une fois les spectateurs installés sur la place de la fête, quatre rois font successivement leur entrée théâtrale. Les dirigeants du festival ordonnent aux gens de s'agenouiller pour le traditionnel salut royal. Près du sanctuaire dressé au milieu de la place, un tambour à cordes et une clochette royale font entendre leurs singulières sonorités. Précédé d'un kapasu au pas de parade, le premier roi s'avance sur la place de la fête, suivi d'un cortège qui va en s'élargissant et passe vers l'arrière au pas de danse, et dont les femmes émettent des sons gutturaux stridents. Juste derrière le roi, les musiciens se font presque bousculer par plusieurs dirigeants du festival qui portent un magnétophone à hauteur d'épaule comme si c'était un reliquaire chaque aspect de Kazanga devant être enregistré, au moins de façon auditive. Cette classe moyenne urbaine ne dispose pas encore de caméras vidéos. Au milieu de la place, quelques membres de l'association Kazanga dansent en se dirigeant vers le roi. Applaudi par la foule, et alors que ses noms honorifiques traditionnels retentissent des hauts-parleurs, le roi prend place dans la loge. Après quelques instants de silence (pendant lesquels d'autres propriétaires de magnétophones à cassettes installent près des musiciens leurs appareils en position d'enregistrement), la foule applaudit le salut royal, après quoi les musiciens agenouillés derrière leurs instruments chantent un hymne de leur répertoire. Cette séquence est répétée pour chaque roi.

Outre l'entrée des rois, le programme du festival Kazanga, distribué sous forme de stencil, contient les points suivants:

- une partie officielle avec l'hymne national zambien (en nkoya), le discours du président de l'association Kazanga et celui du ministre de la Culture;

- des spectacles de danse par divers groupes et danseurs solos afin de présenter, avec l'orchestre d'accompagnement composé d'un xylophone et de tambours, une carte d'échantillons de la culture expressive nkoya (23).

\section{Kazanga et l'Etat}

La médiation visée par Kazanga ne s'oriente que verticalement, c'est-à-dire vers l'Etat, et non pas horizontalement, c'est-à-dire vers les autres groupes ethniques. Le Ministre Junior de la Culture présent, Monsieur Tembo, est originaire de l'Est de la Zambie et, comme $99 \%$ de la population zambienne, ne connaît pas le nkoya. Il y a quelques années, il aurait simplement prononcé son discours en anglais. Mais depuis, beaucoup de choses ont changé en Zambie. Ainsi, avant la cérémonie, le ministre s'est fait dicter par Monsieur D. Mupishi, l'un des 
dirigeants de Kazanga, ancien syndicaliste et marchand de peaux, quelques phrases en nkoya qu'il récite maintenant à l'aide de notes en braille dissimulées dans la poche de son veston. C'est la première fois qu'un représentant de l'Etat s'adresse officiellement aux Nkoya dans leur langue. Le succès est écrasant. Passant rapidement à l'anglais, qui est traduit en nkoya par Monsieur Mupishi, le ministre félicite les dirigeants du festival pour l'excellent accueil qu'ils ont réservé aux hommes politiques et déclare que leur médiation ethnique est une réussite. Il fait appel aux anciens pour qu'ils enseignent aux jeunes 'la signification de Kazanga', et aux jeunes pour qu'ils manifestent de l'intérêt. Cette année, on fête le vingt-cinquième anniversaire de l'indépendance zambienne et le ministre rend gloire à Dieu pour ses bienfaits et au président Kaunda pour sa sagesse.

L'Etat zambien est en faillite et a besoin de tout le soutien possible. Le régime Kaunda touche à sa fin; aux élections démocratiques de 1991, l'UNIP sera battue, après avoir dominé l'Etat pendant près de trente ans, par une coalition démocratique nationale menée par Monsieur $F$. Chiluba. Pendant la nuit qui précéda le festival Kazanga de 1989, la monnaie zambienne a été à nouveau dévaluée de $100 \%$. Les propos religieux doivent masquer le fait que, sur le plan politique, le ministre n'a plus rien à dire. Mais ce n'est pas nécessaire non plus. A la lumière de l'humiliation subie pendant la période coloniale et des défiances premières entre les Nkoya et l'Etat postcolonial, la confirmation inconditionnelle que donne le Ministre Tembo de l'ethnicité nkoya par l'Etat est plus que suffisante pour le public.

Analysons maintenant les détails du processus de médiation ethnique comme il se déroule lors du festival Kazanga.

\section{Sélection et transformation culturelles au festival Kazanga}

L'organisation de service utile charactérise la tache des médiateurs mais ceux-ci pratiquent aussi une sélection et une transformation culturelles qui sont essentielles à la médiation etnique.

En Zambie, comme dans presque tous les pays du monde moderne, la vie publique et la culture politique nationale sont dominées par les médias, en particulier par la radio et la télévision. La médiation ethnique vers le monde extérieur cherche donc à accéder aux médias et, pour ce faire, les festivals sont un moyen éprouvé. Il s'ajoute à cela deux raisons importantes. Premièrement, le district de Kaoma a toujours eu une tradition musicale particulièrement riche (24). Au début du 
dix-neuvième siècle, l'idé d'un orchestre royal à la façon nkoya fut repris de façon permanente par les Lozi. La musique que les Nkoya considèrent de bon droit comme leur étant 'propre' est donc souvent diffusée par les médias zambiens mais comme attribut du pouvoir lozi abhorré. Deuxièmement, la principale expression publique du pouvoir lozi est la cérémonie Kuomboka qui se tient chaque année en avril à l'occasion du déménagement du roi, en chaloupe de gala, de sa résidence d'été à sa résidence d'hiver. Kuomboka jouit depuis un siècle de la grande attention des médias et des hauts dignitaires. Le festival Kazanga se veut être la réponse nkoya à Kuomboka, tout comme l'association Kazanga, avec ses $T$-shirts imprimés pour ses membres, tente d'imiter l'association lozi beaucoup plus riche, plus puissante, plus nombreuse et plus efficace qui rend possible le Kuomboka annuel (25).

Le festival Kazanga est ainsi une nouvelle forme stratégiquement choisie. De quelle manière sélectionne-t-il et transforme-t-il la culture locale existante? Le cadre étroit de cet article m'oblige, hélas, à passer sur le fait que le festival Kazanga se fonde sur un exemple du dix-neuvième siècle qui toutefois se limitait à un seul roi; et nous pouvons seulement constater qu'au milieu de ses rois, le festival moderne - grâce en partie à un sanctuaire construit à neuf pour ses seuls ancêtres - élève Mutondo à une position de séniorité à laquelle il ne peut prétendre traditionnellement. En tant que forme de médiation ethnique, Kazanga vise à présenter une carte d'échantillons de la culture nkoya. A quoi pourrait ressembler une telle carte, vu les formes courantes de culture expressive dans la situation villageoise?

\section{Culture expressive au festival Kazanga}

\subsection{Culture expressive dans la situation villageoise}

La musique et la danse locales nkoya sont depuis deux siècles un modèle pour toute la Zambie occidentale. La richesse dans ce domaine contraste avec la quasi-inexistence des arts plastiques et de l'architecture ornementale (26). La plupart des formes de culture expressive sont liées à des situations spécifiques: initiation des adolescentes, mariage, guérison, transmission du nom, ascension au trône, exécution musicale biquotidienne de l'orchestre royal, fêtes de la guilde des chasseurs. De plus, un répertoire de fête qui suit le goût du jour divertit ceux des villageois qui participent à ces situations dans des rôles non spécialisés. Chaque membre de la communauté a le droit et la compétence de se servir publiquement 
de presque tout le répertoire. Une pure consommation de musique et de danse, excluant toute possibilité de participation active propre, n'existe qu'à l'égard de l'orchestre royal. Dans les autres occasions, de nombreuses voix et textes légèrement divergents retentissent côte à côte en polyphonie, et pour les formes de danse, on peut aussi parler, par analogie, de 'polychorie' (27). Il n'est jamais question de régie, d'orchestration ou de chorégraphie. Les musiciens, les chanteurs et les danseurs se remplacent à leur guise. Les dirigeants, hommes et femmes, veillent seulement à ce que les éventuels rôles solos ne soient pas trop écrasés par la foule. Selon l'endroit et l'époque, ces expressions sont naturellement intégrées dans l'espace social et géographique du village. Elles forment souvent un élément de la vie du village et des individus qui le composent.

Comme structure des activités, le domaine musical et dansant fournit pendant de nombreuses heures, et parfois même plusieurs jours, des situations d'articulation libre et souvent virtuose de l'individu en tant que membre d'un groupe qui, réuni pour une production symbolique, coïncide en grande partie avec le groupe etnique dans lequel ont lieu la production et la reproduction matérielles. Le domaine expressif forme, sans aucune exagération, le pivot de la société villageoise (28).

\subsection{Culture expressive au festival Kazanga}

Que retrouve-t-on de tout cela dans le Kazanga nouveau style? Beaucoup moins que l'on s'y attendrait si l'on considérait ce festival comme étant une expression authentique de la tradition.

La performance en tant que dimension cosmopolite construite de la production symbolique domine le festival. La performance peut se décrire comme suit: c'est une activité spécialisée, structurée et standardisée dans les moindres détails par une régie; détachée dans l'espace et le temps du contexte usuel de production et de reproduction matérielles; et présentant une séparation très nette entre (a) les dirigeants, (b) les producteurs directs c'est-à-dire les exécutants, et (c) la foule des consommateurs réduits à l'incompétence et à la non participation productives.

Une telle forme de production nie les caractéristiques du domaine expressif dans la société villageoise. Elle offre une matrice dans laquelle des éléments détachés sont insérés et remplacés à souhait; ces éléments sont transformés en objets et consommés pour acquérir, parmi d'autres performances similaires, une valeur marchande dans le monde extérieur considéré comme un marché de produits 'performatifs'. Les éléments composant la performance sont empruntés à un 
idiome local mais fonctionnent dans un autre contexte et d'une manière tellement différente que l'idée de continuité par rapport à la tradition n'est pas soutenable.

Examinons de plus près les trois rôles de dirigeant, d'exécutant et de spectateur, à commencer par ce dernier.

Sous les instructions assourdissantes diffusées par les hauts-parleurs, les spectateurs debout le long de la place de la fête supportent assez bien leur passivité. Ils acclament avec enthousiasme l'entrée des rois et la plupart des spectacles, et beaucoup se balancent involontairement au rythme de la musique. Seules quelques femmes âgées revendiquent leur droit de danser et de chanter à pleins poumons. Leurs mouvements de danse sont vifs et impliquent tout le corps sans aucune gêne.

Ensuite les exécutants. Quelques-uns seulement des quinze spectacles présentés le jour du festival sont préparés par de vrais villageois, provenant notamment de la région de Kahare. Ils transmettent leur culture expressive avec un minimum de régie et de chorégraphie, dans leurs habits de tous les jours, et la plupart pieds nus. Mais leur participation ne tient pas à une raison évidente qui se serait imposée dans l'espace et le temps de leur village, mais au fait qu'ils sont cooptés par des médiateurs ethniques. Dans ces périodes difficiles, la perspective d'un revenue monétaire est alléchante, et ils sont profondément déçus après le festival de rentrer chez eux avec bien peu: de quoi s'acheter un paquet de cigarettes.

Les autres exécutants sont des danseurs solos qui incarnent un bouffon de cour, un chasseur ou un guerrier traditionnels, et sont couverts de parures que l'on n'avait pu admirer depuis bien longtemps, ainsi que quatre petits groupes de danse féminins: deux originaires d'une école de village, un constitué de membres féminins de l'association Kazanga à Lusaka, et un autre composé de deux jeunes villageoises interprétant la danse de la kankanga qui clôt l'initiation des adolescentes. Ces dernières sont conduites sur la piste de danse, courbées sous une couverture selon la coutume, mais elles ne sont nettement plus des kankangas: leurs seins sont bien développés et recouverts, contre la tradition, de soutien-gorges blancs; ces femmes n'ont rien de la grâce timide et craintive d'une débutante, mais elles agitent avec élégance des petits foulards blancs à la fin de leur danse. Pendant la danse, les citadines se reconnaissent facilement: elles portent des chaussures, leurs cheveux sont coûteusement coiffés, certaines ont des lunettes de soleil et toutes portent au-dessus du pagne, qui est une concession incontournable au goût villageois, le même $T$-shirt blanc imprimé avec l'inscription 
'KAZANGA 1989 - NKOYA CULTURAL CEREMONY'. Leur inhibition motrice renvoie aux conceptions de la classe moyenne occidentale et au christianisme cosmopolite: ceci reflète une tentative indéniable de construire une culture ethnique acceptable par une société plus large, dans ce sens aussi qu'elle ne confirme pas les stéréotypes de 'paganisme' et de 'primitivité'. Les femmes de chaque groupe de danse sont toutes habillées de la même façon et s'efforcent de faire toutes ensemble les mêmes mouvements et pas de danse, selon des figures géométriques en forme de cercle et de ligne. En conséquence, leur performance acquiert une unité sans relief, une prédictibilité et une pauvreté de forme en opposition flagrante avec leur culture expressive traditionnelle. La danse des citadines est coordinée par un danseur qui, certes, porte des vêtements féminins, une perruque blonde en nylon (!) et des claquettes aux jambes mais qui, à nouveau chose impensable dans la situation villageoise, ne cesse de mettre l'accent sur son autorité masculine sur les femmes qui dansent et chantent. Il danse aussi avec les autres groupes féminins, même avec les fausses kankangas.

Au niveau des exécutants, Kanzanga exprime des oppositions qui, malgré la tentative d'unité pan-nkoya, font éclater le groupe ethnique en camps opposés: oppositions entre styles de vie citadin et rural, entre classes, entre hommes et femmes et entre religion autochtone et christianisme (29). Ces oppositions sont, chose étonnante, à peine masquées symboliquement; au contraire, la subordination au groupe dominant (citadins, classe moyenne, hommes) est rendue plus explicite encore. Une interprétation en termes de 'mauvaise conscience masquante' ne peut avoir sa place ici.

Enfin les dirigeants. Nous en avons déjà rencontré certains en train de danser en $T$-shirt, de prononcer des discours, de diriger le programme du festival et de donner au microphone des instructions au public. Outre le $T$-shirt, le costume-cravate est leur parure caractéristique. Toujours reconnaissables comme une catégorie à part, ils délibèrent continuellement et sans signes de friction, près du microphone, avec les dignitaires à la cour de Mutondo en tenue similaire auxquels ils sont souvent apparentés. Leur comportement à l'égard des hommes politiques nationaux est moins désinvolte, et beaucoup retombe sur les épaules de Monsieur Mupishi qui accompagne les invités de haut rang et souffle des formules diplomatiques à ses coadministrateurs. Aucun des leaders cravatés ne se laisse tenter à danser ou à faire le salut royal, - leur rôle de médiateur semblant exiger une distance supérieure par rapport au produit offert. 
7.3. La dimension performative comme monnaie d'échange vers le monde extérieur

La dimension performative de Kazanga est une formule cosmopolite importée avec une force politique et idéologique considérable, depuis le début du siècle, dans le district de Kaoma, par la mission et l'enseignement et promue ensuite par l'Etat postcolonial à l'occasion de la célébration des fêtes nationales, de la présentation des spectacles agricoles, etc. Rien d'étonnant donc à ce que cette formule facilite la médiation vers l'Etat qui s'appuie lourdement, pour sa propre légitimation, sur la production culturelle performative (30). La performance formalisée, contact unilatéral qui ne s'enracine plus dans une pratique de production et de reproduction matérielles mais qui réduit le groupe-cible à une incompétence passive, n'est qu'une métaphore de la relation entre l'Etat moderne et le citoyen. La formule performative parvient quotidiennement aux consommateurs zambiens par l'intermédiaire de la radio et de la télévision et va des spectacles de la Troupe nationale de danse à la série télévisée nord-américaine 'Dallas' en passant par les hit-parades internationaux de musique pop. C'est la formule de la culture de masse actuelle, propagée à l'échelle mondiale par les médias électroniques. C'est cette partie de l'expérience africaine actuelle qui rejoint et même correspond à notre expérience propre, similairement structurée en tant que consommateurs de médias et de culture occidentale sur un marché de produits symboliques commercialisés, dirigés et aliénés, et parmi lesquels on trouve d'ailleurs de plus en plus de produits africains modernes.

Les ressources électroniques (représentées à Kazanga par les hauts-parleurs et les magnétophones), trahissent le mode de production capitaliste dominant à l'échelle mondiale dont les caractéristiques essentielles sont la séparation entre les producteurs directs et leur produit, l'aliénation qui en dérive, le marché comme base principale de formation des valeurs et l'accent mis sur la standardisation et l'interchangeabilité - comme s'il s'agissait d'un produit industriel ou d'un ouvrier. Ces caractéristiques sont si évidentes à Kazanga (31) que le festival doit être vu comme une médiation non seulement entre la communauté locale et l'Etat, mais aussi entre la production non capitaliste de cette communauté et le mode de production capitaliste. 


\section{Conclusion}

Un chercheur non préparé qui, lors de sa recherche sur le terrain dans le district de Kaoma, se heurterait au festival Kazanga, serait sans doute enclin à considérer le phénomène comme un élément intégrant de la culture locale et négligerait les nombreuses implications politiques et culturelles du processus de médiation ethnique. Cependant, les études anthropologiques faites dans le district au cours des vingt dernières années offrent une pierre de touche pour ce qui est présenté aujourd'hui comme étant la 'culture nkoya traditionnelle'. Kazanga ne semble en aucun cas être l'expression d'une identité culturelle traditionelle bien définie. Il représente l'abandon des identités locales diffuses en faveur de la construction d'une identité ethnique valant monnaie d'échange vers le monde extérieur où ses produits sont reconnaissables.

Mon analyse a montré que le festival, dont personne ne nie le caractère extrêmement récent, est moins basé sur une légitimité fondée sur le passé que sur la médiation de la culture locale vers le monde extérieur actuel, où cette culture est radicalement transformée et où de nouvelles inégalités sont créées et accentuées.

L'analyse de Kazanga n'offre pas seulement une illustration d'un modèle de sélection et de transformation culturelles dans un contexte de médiation ethnique - modèle qui semble avoir une applicabilité considérable, des mouvements afrikaner et zoulou sud-africains (32) aux modèles d'autorité de la société multiculturelle néerlandaise (33). Kazanga soulève une question très importante dans le développement actuel vers une société mondiale. Reste-t-il au bien culturel des sociétés périphériques impuissantes du tiers monde un autre avenir que celui de s'insérer dans le monde extérieur, plus ou moins comme folklore, sous une dimension aliénée? Le transfert culturel implique nécessairement un détachement à l'égard du contexte initial, mais c'est la seule manière non seulement de documenter et d'expliquer les acquisitions inestimables des innombrables cultures du tiers monde, mais aussi de les incorporer comme partie intégrante du patrimoine humain.

Un facteur important de l'attrait que ces cultures lointaines exercent sur nous, l'une des raisons pour lesquelles chaque génération d'anthropologues occidentaux repart vers l'Afrique, est l'espoir de faire l'expérience d'une culture totalement différente dans son contexte, sa cohésion et sa signification, pour la gloire de la culture humaine comme totalité et pour l'enrichissement de notre existence. Une 
telle tentative est souvent condamnée comme étant romantique et égoïste et se concilie mal avec la subordination qui détermine depuis des siècles les relations Nord-Sud. Néanmoins, elle a produit une littérature ethnographique qui réussit souvent à servir de médiateur entre la communauté locale étudiée et la société cosmopolite. Au cours du reportage, l'ethnographe se distancie de son expérience directe sur le terrain - tout comme Kazanga se distancie du domaine expressif comme il fonctionne au niveau du village (34). Y a-t-il des conditions sous lesquelles, à Kazanga, dans d'autres formes de traduction culturelle et dans l'ethnographie, ce processus peut se dérouler sans jeter le bébé avec l'eau du bain? De ce point de vue, mon analyse de Kazanga est pessimiste.

La reconnaissabilité du propre comme propre est cependant un processus flexible pour lequel le propriétaire n'a pas de compte à rendre aux autres. L'anthropologue constate que la médiation ethnique fournit un produit culturel fondamentalement différent de 'la chose réelle' - mais, qui déniera aux participants le droit d'être satisfaits du produit transformé, de s'y reconnaître, et de l'envoyer dans le monde extérieur? La dimension performative, et plus généralement chaque médiation dans laquelle la structure du monde extérieur cosmopolite est déterminante, offre des possibilités de négociation avec l'Etat et l'économie mondiale qui, vu la dominance de cette dernière, sont peut-être la seule chance de survie pour les éléments culturels locaux (35).

L'enthousiasme non déguisé avec lequel les villageois acclament les rois à leur entrée, et avec lequel ils se bousculent pour donner de l'argent même aux fausses kankangas, suggère qu'ils seraient en désaccord avec la partie de mon analyse de Kazanga qui met l'accent sur les violences faites à leur logique culturelle propre. Leur flexibilité à l'égard des canons traditionnels, leur confiance implicite dans le fait que ce qui compte vraiment n'est pas nécessairement perdu malgré la sélection, la transformation et le poids des nouvelles inégalités, devraient encourager les anthropologues dans leurs tentatives de médiation intellectuelle des sociétés locales du tiers monde.

Les phénomènes ethniques dominent le monde d'aujourd'hui et de demain; la lecture des journaux offre, sur ce point, de nombreux exemples. Dans une Europe occidentale en marche vers une unité politique et économique, notre langue et notre culture historique nationales prendront, elles aussi, le caractère d'une expression ethnique à l'intérieur d'un ensemble plus grand. Comment avoir prise sur ces processus mondiaux qui sont des manifestations multiples des principaux thèmes présentés ici: le paradoxe entre l'ascriptif invariable et le manipulatif mobilisant; et la formation de pouvoir et d'identité par médiation entre les 
symboles locaux d'une part et l'Etat et l'économie mondiale de l'autre? L'anthropologie peut largement contribuer à notre compréhension par:

- une connaissance détaillée et approfondie des sociétés locales dans leurs tentatives pour entrer en contact avec le monde extérieur;

- la recherche d'un équilibre entre des théories abstraites et globales, et la leçon qui peut être tirée, avec beaucoup plus de difficultés, du matériel ethnographique spécifique;

- la prise de conscience qu'à côté de l'économie politique (36) et de l'histoire, le domaine symbolique joue un rôle capital dans les phénomènes ethniques et doit être approché avec les méthodes appropriées;

- la prise de conscience toujours renouvelée et vérifiée par la pratique de l'observation participante, du dialogue et de l'identification entre le chercheur et l'objet de sa recherche, qui ouvre la voie vers une science humaine vraiment humaine.

Ces perspectives peuvent s'appliquer de façon fructueuse à l'Afrique - notamment à l'Afrique australe, où, par exemple, la lutte pour un Etat post-ségrégationniste en Afrique du Sud, l'hégémonie des Tswana au Botswana (37) et le jeu d'équilibre entre les identités shona, ndebele et européennes au Zimbabwe (38) requièrent un terrain d'étude complexe pour le développement des thèmes que je vous ai présentés ici à très petite échelle.

Traduit du néerlandais par Evelyne Codazzi

\section{Notes}

1. Forme abrégée du discours inaugural prononcé par l'auteur le 20 mars 1992 à l'occasion de son entrée en fonctions comme professeur extraordinaire sur mandat de la fondation Afrika-Studiecentrum à la chaire 'Ethnicité et idéologie dans les processus de développement dans le tiers monde' de la Faculté des Sciences socio-culturelles de l'Université libre d'Amsterdam. Le financement de la traduction en français a été pris en charge par le Département d'Anthropologie et de Sociologie de développement, Université libre d'Amsterdam.

2. Voir Génèse 2:19.

3. Fardon (1987) nie en revanche l'existence d'universaux dans l'étude sur l'ethnicité.

4. Par exemple Lévi-Strauss (1962).

5. Gutkind (1970); Helm (1968); Godelier (1973), p. 93-131: 'Le concept de tribu: Crise d'un concept ou crise des fondements empiriques de l'anthropologie?'; Ranger (1982); Vail (1989a, 1989b); Bayard (1989) notamment p. 65-86: 'Le théâtre d'ombres de l'ethnicité'. 
6. Il est impossible de rendre compte ici de l'immense littérature qui couvre les diverses disciplines traitant de l'identité. L'un des auteurs les plus influents est le psychiatre Erikson (notamment 1968). D'importantes études d'anthropologie sur l'identité ethnique se trouvent notamment in de Vos \& Ramanucci-Ross (1975); Horowitz (1975); Jacobson-Widding (1983). Pour notre sujet zambien, voir Ethos and identity (Epstein 1978), qui se distancie de l'accent mis sur la simple classification dans l'étude sur l'ethnicité de son proche collègue Mitchell (1956, 1974). L'approche magistrale de Blacking (1983) accorde une large attention à la culture expressive. Pour une contribution française inspirante, voir Amselle (1990).

7. Pour une opinion similaire, voir Uchendu (1975): 265.

8. D'anciens chercheurs se sont laissés séduire par cet aspect et ont décrit l'ethnicité en termes d'identité de base ('primordial identity'), façon de voir avec laquelle Doornbos (1972) en finit une fois pour toutes.

9. L'anthropologie marxiste traite de la médiation entre de telles relations sociales structurées, fondamentalement différentes, en termes de liaison ou d'articulation des modes de production, voir Geschiere (1982); van Binsbergen \& Geschiere (1985a); et références ibidem. Bien que l'étude sur l'ethnicité montre que le domaine symbolique ne peut être placé de façon convaincante dans une relation subordonnée à l'égard de la production et de la reproduction, la perspective de l'articulation de modes de production n'en est pas moins inspirante - voir van Binsbergen (1985).

10. Le rôle central de ce type de médiateurs est commenté dans une immense littérature anthropologique, dans laquelle Barth (1966) occupe une place classique.

11. Le monde extérieur dans la médiation ethnique ne comprend pas seulement l'Etat. Peel (1986) décrit l'identité yoruba comme un projet du dix-neuvième siècle dans lequel, tout comme pour les Nkoya, un ancien chef d'Eglise jouait un role capital. Vail (1989b) mentionne, outre les hommes politiques et les chefs d'Eglise locaux, les chercheurs scientifiques comme médiateurs dans de nombreux processus d'ethnisation en Afrique du Sud; voir Papstein (1989); van Binsbergen (1985). Le processus de médiation est également un thème chez Ranger (1982). De récentes études d'Afrikaners en Afrique du Sud ont mis en lumière le rôle de médiateur ethnique de l'écrivain - et il existe à cet égard de nombreux parallèles ailleurs en Afrique, comme par exemple Okot p'Bitek, défenseur de l'ethnicité acholi en Ouganda (van de Werk 1980).

12. Voir Edelstein (1974); Saul (1979); Kahn (1981); van Binsbergen (1985). Le point de vue marxiste domine l'approche de l'ethnicité parmi la population noire sud-africaine. La lutte contre l'Etat ségrégationniste comme manipulateur ou même créateur de l'ethnicité noire conduit à la négation de l'ethnicité comme variable indépendante; elle ne peut être alors qu'une conscience de classe pervertie (par exemple Simons \& Simons (1969); Mafeje (1971); Phimister \& van Onselen (1979). Ces dernières années, un certain changement est apparu dans cette attitude politiquement compréhensible mais scientifiquement trop réductionniste (par exemple Beinart 1988 qui, dans la biographie détaillée d'un migrant, met aussi en cause les stratégies ethniques parmi la population noire).

13. Des études sur le terrain ont été menées en 1972-1974 dans le district de Kaoma et à Lusaka parmi des migrants originaires de Kaoma, ainsi que lors de brefs séjours financés par l'Afrika-Studiecentrum en 1977, 1978, 1981 et 1989. J'ai bénéficié en 1974-1975 d'une année d'étude subventionnée par la fondation WOTRO. Pour la société locale et son histoire, voir van Binsbergen (1981, 1985, 1987a, 1991a, 1991b, 1992a); van Binsbergen \& Geschiere (1985b notamment p. 261-270, 'production at a Zambian chief's court'); et références ibidem. 
14. Sur les Lozi, surtout connus par les travaux de Max Gluckman, voir par exemple Prins (1980) et les abondantes références ibidem.

15. Sur les Luvale, voir Papstein (1989) et références ibidem.

16. Certaines indications (dont je ne peux juger la valeur linguistique) tendent à prouver que le nom Nkoya est plus ancien encore; que c'est une altération du nom 'Kola' qui désignait le coeur de la région lunda, berceau de nombreuses dynasties dans le Sud de l'Afrique centrale.

17. Dans le Nord du Barotseland, le groupe luvale a pu se soustraire en 1940 à l'autorité lozi et former son propre district, placé directement sous l'Etat central; cf. (Papstein 1989); pour l'influence de ce processus sur l'ethnicité nkoya, voir van Binsbergen (1992a): 39.

18. En fait: 'Andrew Murray Memorial Mission', appelée plus tard 'Africa Evangelical Fellowship' dont l'église missionnaire est devenue indépendante du point de vue de l'organisation sous le nom de 'Evangelical Church of Zambia'.

19. Testamenta (1952).

20. Anonyme, sans date; et l'ouvrage principal de Shimunika: Likota lya Bankoya, rédigé par moi en nkoya et en anglais: van Binsbergen (1988a, 1992a). La traduction anglaise dans l'édition de 1992 est de M. Malapa, président de l'association Kazanga jusqu'en 1990, et de moi-même. 21. Caplan (1970); Molteno (1974).

22. En 1989, la loge d'Etat était occupée notamment par: Monsieur J. Mulimba, Ministre du Travail, du Développement social et de la Culture, membre du Comité central de l'UNIP; Monsieur L. Tembo, Ministre Junior de la Culture; Monsieur J. Kalaluka, simple particulier, jusqu'en 1988 membre du parlement pour le district et ministre des Affaires économiques; et Madame S. Mulenga, épouse du gouverneur du district de Kaoma empêché pour raison de santé. 23. La liste des danseurs solos inscrits au programme comprenait Mwene Mutondo dont la danse royale devait fournir l'un des clous du festival, mais cet élement a été abandonné, soi-disant pour raisons de santé, mais sans doute aussi pour ne pas propager l'hégémonie Mutondo plus explicitement encore que ce n'était déjà le cas (voir paragraphe 7).

24. Voir Brown (1984); Kawanga (1978); et mes publications.

25. Voir Brown (1984); van Binsbergen (1987b); et références ibidem.

26. D'autres recherches sont nécessaires pour déterminer si cette absence d'arts plastiques est surtout une conséquence des influences iconoclastes du christianisme et des mouvements d'anti-sorcellerie actives en pays nkoya depuis le début du vingtième siècle. Un éventuel art plastique ancien aurait surtout concerné des objets se rapportant au culte des ancêtres et à la magie. Les bâtons d'ancêtres dans le sanctuaire Mutondo sont, selon mon expérience, uniques dans le district, mais il en existe ailleurs dans le Nord-Ouest de la Zambie - ils seraient en fait largement répandus sur tout le continent africain.

27. Du grec polu, 'beaucoup' et choros, 'danse'.

28. Voir van Binsbergen (1991a).

29. Une opposition théorique évidente et indéniable dans la société villageoise du district de Kaoma mais qui ne semble pas exprimée par Kazanga est celle entre les anciens (du sexe masculin) et les jeunes. Une réflexion plus approfondie s'impose sur ce point.

30. T.O. Ranger et R.P. Werbner ont organisé en 1986 à Manchester une conférence inspirante sur cet aspect des transformations culturelles dans l'Etat africain postcolonial: 'Culture et conscience dans l'Afrique du Sud'. Certaines contributions ont été publiées entre-temps dans le Journal of Southern African Studies; voir aussi Kaarsholm $(1989,1990)$.

31. Par exemple: la régie égalisante du vêtement et de la motorique, la répression de la polyphonie et de la polychorie, la rétribution en argent et la réduction de la plupart des personnes présentes en consommateurs incompétents. 
32. Sur les Afrikaners, voir Moodie (1975); February (1991); Giliomee (1989, 1989); Hofmeyr (1988); sur les Zoulous et les Inkathas, cf. Coquerel (1989); Maré \& Hamilton (1987); Marks (1989); Schlemmer (1980).

33. Les études sur la société multiculturelle néerlandaise accordent surtout de l'attention aux médiateurs ethniques n'appartenant pas aux groupes ethniques en articulation mais au monde extérieur (hollandais) - les 'gérants' (Köbben 1983; van Borselen 1985); pour les médiateurs originaires des groupes ethniques, voir van Wetering (1991); van der Burg \& van der Veer (1986); Koot \& Venema (1985); Bruin (1985) dans l'ouvrage passionnant de Bovenkerk et al. (1985).

34. Voir Clifford \& Marcus (1986); Geertz (1988). J'ai intensivement étudié ces dernières années la notion de médiation anthropologique et culturelle entre la communauté locale et le monde extérieur cosmopolite, ainsi que les problèmes méthodologiques, esthétiques, éthiques et politiques qui lui sont liés; voir van Binsbergen (1985, 1985, 1988b, 1988c), van Binsbergen \& Doornbos (1987).

35. Le dilemme esquissé se trouve à la base de l'affaire Rushdie, écrivain condammné à mort pour une médiation (dans ce cas littéraire) de l'islam que certains musulmans considèrent comme étant trop transformative; Rushdie lui-même écrit: 'Les versets sataniques est un plaidoyer pour un changement-par-fusion, pour un changement-par-liaison. C'est une ode à notre moi bâtard. Tout au long de l'histoire de l'humanité, les apôtres de la pureté, ceux qui prétendaient posséder une explication totalisante, ont fait des ravages parmi des gens dont la seule faute était leur incertitude morale. Tout comme des millions d'autres gens, je suis un bâtard de l'histoire. Il est bien possible que nous tous, noirs, bruns et blancs, nous nous entremêlions, comme dit l'un de mes personnages, comme les saveurs à la cuisson' (Rushdie 1990: 12).

36. Par exemple Shaw (1986); Rothschild \& Olorunsola (1983); Bayart (1989). L'étude abstraite de Shaw est un exemple de ce qu'un projet ethnographique plus modeste peut nous faire éviter; sa généralisation, à savoir que l'expression dominante dans les systèmes politiques africains est formée en période de croissance économique par l'ethnicité et en période de déclin économique par la lutte des classes, semble en contradiction avec ce que nous a appris la simple observation participante.

37. En accord avec l'image officielle du Botswana comme Etat mono-ethnique, peu d'études ont été faites sur les processus ethniques dans ce pays - hiatus que j'espère pouvoir combler dans les années qui viennent. Ebauches dans Picard (1987); Wilmsen (1989); van Binsbergen (1991b). Comme préparation, $\mathrm{j}$ 'ai étudié le développement de perspectives sur les relations raciales, le vécu culturel public et la relation entre l'Etat et la société civile au Botswana (van Binsbergen 1990, 1991c, 1992b).

38. Par exemple Ranger (1989); Schutz (1990).

\section{Bibliographie}

AmSElle, J.L., 1985, Logiques métisses; Anthropologie de l'identité en Afrique et ailleurs, Paris: Payot.

ANONYME [J.M. Shimunika], sans date. Muhumpu wa byambo bya mwaka - Nkoya, sans lieu [Luampa, Mankoya]: sans nom [South African General Mission], photocopie, collection de l'auteur. 
BARTH, F., 1966, Models of social organization, Londres: Royal Anthropological Institute of Great Britain and Ireland, Occasional Papers no. 23.

BAYART, J.F., 1989, L'Etat en Afrique: La politique du ventre, Paris: Fayard.

BEINART, W., 1988, 'Worker consciousness, ethnic particularism and nationalism: The experience of a South African migrant, 1930-1960' in Marks \& Trapido 1988: 286-309.

BLACKING, J., 1983, 'The concept of identity and folk concepts of self: A Venda case study' in Jacobson- Widding 1983: 47-65.

BOVENKERK, F., K. BRUIN, L. BRUNT \& H. WOUTERS, 1985, Vreemd volk, gemengde gevoelens, Meppel/Amsterdam: Boom.

BROWN, E.D., 1984, 'Drums of life: Royal music and social life in Western Zambia', thèse de doctorat, Université de Washington, Ecole de musique; Microfilms de l'Université, Ann Arbor.

BRUIN, K., 1985, 'Het Oostersch feest' in Bovenkerk et al. 1985: 155-259.

CAPLAN, G., 1970. The elites of Barotseland 1878-1969, Londres: Hurst.

ChrÉtIEN, J.-P., \& G. PRUNIER, réd., 1989, Les etnies ont une histoire, Paris: Karthala/Agence de Coopération Culturel et Technique (ACCT).

ClifFORD, J., \& G. MARCUS, éd., 1986, Writing culture, Berkeley, University of Califomia Press.

COQUEREL, P., 1989, 'Les Zulu dans l'Afrique du Sud contemporaine (Le mouvement Inkatha Yenkululeko Yesizwe - Ethnicité et lutte de liberation)' in Chrétien \& Prunier 1989: 417-425.

de Vos, G., \& L. RomanucCi-Ross, réd., 1975, Ethnic identity; Cultural continuities and change, Palo Alto: Mayfield.

DOORNBOS, M.R., 1972, 'Some conceptual problems concerning ethnicity in integration analysis', Civilisations, 22, 2: 268-83.

EDELSTEIN, J.C., 1974, 'Pluralism and Marxist perspectives on ethnicity and nation-building', in W. Bell \& W.E. Freeman, réd. Ethnicity and nation-building: Comparative, international and historical perspectives, Beverly Hills/Londres: Sage p. 45-57.

EPSTEIN, A.L., 1978, Ethos and Identity: Three studies in ethnicity, Londres: Tavistock.

FARDON, R., 1987, 'African ethnogenesis: Limits to the comparability of ethnic phenomena', in L. Holy, réd. Comparative anthropology, Oxford: Basil Blackwell, p. 168-188.

February, V., 1991, The Afrikaners of South Africa, Londres/Boston: Keagan Paul International.

GEERTZ, C., 1988, Works and lives, Stanford: Stanford University Press.

GESCHIERE, P.L., 1982, Village communities and the state, Londres/Boston: Keagan Paul International.

GIIOMEE, H., 1989, 'The beginnings of Africaner ethnic consciousness, 1850-1915', in Vail 1989a: 21-54.

GODELIER, M., 1973, Horizon, trajets marxistes en anthropologie, Paris: Maspero.

GUTKIND, P.C.W., réd., 1970, The passing of tribal man in Africa, Leiden: Brill.

HELM, J., red., 1968, Essays on the problem of tribe: Proceedings of the 1967 Spring meeting of the American Ethnological Society, Seattle/Londres: University of Washington Press.

HOFMEYR, I., 1988, 'Building a nation from words: Afrikaans language, literature and ethnic identity, 1902-1924', in Marks \& Trapido 1988: 95-123.

HoROwITZ, D.L., 1975, 'Ethnic identity', in Glazer, N., \& D.P. Moynihan, réd., Ethnicity: Theory and experience, Cambridge (Mass.)/Londres: Harvard University Press, p. 111-140.

JACOBSON-Widding, A., réd., 1983, Identity: Personal ans socio-cultural, Uppsala. 
KAARSHOLM, P., 1989, 'Quiet after the storm', African languages and cultures, 2, 2: 175-202.

KAARSHOLM, P., 1990, 'Mental colonization or catharsis', Jourmal of Southern African Studies, 16, 2: 246-75

KAHN, J., 1981, 'Explaining ethnicity: A review article' Critique of Anthropology, 4, 16: 43-52.

KAWANGA, Davidson, 1978, 'Nkoya songs as taped by Wim van Binsbergen: translations and notes', manuscript, collection de l'auteur.

KOOT, W., \& P.U. VENEMA, 1985, 'Etnisering en etnische belangenbehartiging bij Surinamers: een nieuw stijgingskanaal', Migrantenstudies, 1, 1: 4-16.

KÖBBEN, A.J.F., 1983, De zaakwaarnemer, discours inaugural à l'Université Erasme de Rotterdam, Deventer: Van Lochum Slaterus.

LÉVI-STRAUSS, C., 1962, Le totémisme aujourd'hui, Paris: Presses Universitaires de France.

MAFEJE, A., 1971, 'The ideology of tribalism', Journal of Modern African Studies, 9: 253-61.

MARE, G., \& G. HAMIT TON, 1987, An appetite for power: Buthelezi's Inkatha and the politics of 'loyal resistance', Johannesburg: Ravan Press.

MARKS, S., \& S. TRAPIDO, réd., 1988, The politics of race, class and nationalism in twentieth-century South Africa, Londres/New York: Longman, réimpression de la première édition de 1987.

MARKS, S., 1989, 'Patriotism, patriarchy and purity; Natal and the politics of Zulu ethnic consciousness', in Vail 1989a: 215-240.

MrTCHELL, J.C., 1956, The Kalela dance: Aspects of social relationships among urban Africans in Northern Rhodesia, Manchester: Manchester University Press.

MrTCHELl, J.C., 1974, 'Perceptions of ethnicity and ethnic behaviour: An empirical exploration', in A. Cohen, réd., 1974, Urban ethnicity, Londres: Tavistock, p. 1-35.

Molteno, R., 1974, 'Cleavage and conflict in Zambian politics' in W. Tordoff, réd., Politics in Zambia, Manchester: Manchester University Press, p. 62-106.

MOODIE, T.D., 1975, The rise of Afrikanerdom, Berkeley/Los Angeles/Londres: University of California Press.

OKoT P'BITEK, 1980, Lied van Lawino en Lied van Ocol, translated into Dutch by W. van Binsbergen \& A. van Rijsewijk, Maasbree: Corrie Zelen.

PAPSTEIN, R.J., 1989, 'From ethnic identity to tribalism: The Upper Zambezi region of Zambia $1830-1981$ ', in Vail 1989a: 372-394.

PEEL, J.D.Y., 1989, 'The cultural work of Yoruba ethnogenesis', in E. Tonkin, M. McDonald \& M. Chapman réd., 1989, History and ethnicity, Londres/New York: Routledge, p. 198-215

PHIMISTER, I.R. \& C. van ONSELEN, 1979, 'The political economy of tribal animosity: A case study of the 1929 Bulawayo "faction fight" ', Joumal of Southern African Studies, 6,1:1-43.

PICARD, L.A., 1987, The politics of development in Botswana: A model for success?, Boulder \& Londen: Lynne Rienner.

PRINS, G., 1980, The hidden hippopotamus, Cambridge: Cambridge University Press.

RANGER, T.O., 1982, 'Race and tribe in Southern Africa: European ideas and African acceptance', in R. Ross, réd., Race and colonialism, La Haye: Nijhoff, p. 121-42.

RANOER, T.O., 1989, 'Missionaries, migrants and the Manyika', in Vail 1989a: 118-150.

REEFE, T.Q., 1981, The rainbow and the kings: A history of the Luba empire to 1891, Berkeley \& Los Angeles: University of Califiornia Press.

ROTHCHLD, D., \& V.A. OlORUNSOLA, réd., 1983, State versus ethnic claims: African policy dilemmas, Boulder (Col): Westview. 
RUSHIE, S., 1990, Is er dan niets meer heilig: Over religie en literatuur, Utrecht/Anvers: Veen. SAUL, J., 1979, The state and revolution in eastern Africa, New York: Monthly Review Press. SCHLEMMER, L., 1980, 'The stirring giant: Observations on the Inkatha and other Black political movements in South Africa' in R.M. Price \& C.G. Rosberg, réd., The apartheid regime: political power and racial domination, Berkeley: University of California p. 99-126.

SCHUTZ, B.M., 1990, 'Political change and the management of ethnic conflict in Zimbabwe', in J.V. Montville, réd., Conflict and peacemaking in multiethnic societies, Lexington/Toronto: Heath (Lexington Books) p. 433-447.

SHAW, T.M., 1986, 'Ethnicity as a resilient paradigm for Africa: From the 1960s to the 1980s', Development and Change, 17, 1: 587-605.

SimONS, H.J. \& R.E. SimONS, 1969, Class and colour in South Africa 1850-1950, Harmondsworth: Penguin Books.

TESTAMENTA, 1952, Testamenta ya yipya/Nyimbo [Nkoya New Testament and Psalms], Londres: British and Foreign Bible Society.

UCHENDU, V.C., 1975, 'The dilemma of ethnicity and polity primacy in Black Africa', in de Vos \& Romanucci-Ross 1975: 265-275.

VAIL, L., réd., 1989a, The creation of tribalism in Southern Africa, Londres/ Berkeley \& Los Angeles: Currey/University of California Press.

VAIL, L. 1989b, 'Ethnicity in Southern African history', in Vail 1989a: 1-19.

van BinSBERGEN, W.M.J., 1981, Religious change in Zambia, Londres/Boston: Kegan Paul International.

van BINSBERGEN, W.M.J., 1984, 'Kann die Ethnologie zur Theorie des Klassenkampfes in der Peripherie werden?', Osterreichische Zeitschrift für Soziologie, 9, 4,: 138-48.

van BINSBERGEN, W.M.J., 1985, 'From tribe to ethnicity in Western Zambia: The unit of study as an ideological problem', in Binsbergen \& Geschiere 1985a: 181-234.

van BINSBERGEN, W.M.J., 1987a, 'De schaduw waar je niet overheen mag stappen: Een westers onderzoeker op het meisjesfeest van de Zambiaanse Nkoya', in Binsbergen \& Doombos 1987,: 139-181.

van BINSBERGEN, W.M.J., 1987b, 'Chiefs and the state in independant Zambia: Exploring the Zambian National Press', Journal of Legal Pluralism and Unofficial Law, no. 25-26, 1987: 139-201.

van BINSBERGEN, W.M.J., 1988a, réd., J. Shimunika's Likota lya Bankoya: Nkoya version, Research report no. 31B, Leiden: Afrika-Studiecentrum.

van BINSBERGEN, W.M.J., 1988b, 'Reflections on the future of anthropology in Africa', in C. Fyfe, réd., African futures, Edimbourgh: Centre of African Studies, p. 293-309.

van BINSBERGEN, W.M.J., 1988c, Een buik openen, Haarlem: In de Knipscheer.

van BINSBERGEN, W.M.J., 1990, 'African Independent churches and the state in Botswana', contribution à la conférence 'Power and prayer', Instituut voor de Studie van Politiek en Religie, Vrije Universiteit, Amsterdam, décembre 1990.

van BINSBERGEN, W.M.J., 1991a, 'De chaos getemd? Samenwonen en zingeving in modern Afrika', in H.J.M. Claessen, réd., De chaos getemd?, Leiden: Faculteit der Sociale Wetenschappen, Rijksuniversiteit, Leiden, p. 31-47.

van BINSBERGEN, W.M.J., 1991b, 'Minority language, ethnicity and the state in two African situations: the Nkoya of Zambia and the Kalanga of Botswana', contribution à la 'Conférence on African Languages, development and the state', SOAS, Londres, avril 1991; sous presse in R. Fardon \& G. Furniss, réd., Language and languages [titre provisoire]. 
van BiNSBERGEN, W.M.J., 1991c, 'Becoming a sangoma: Religious anthropological field-work in Francistown, Botswana', Journal on Religion in Africa, 21, 4: 309-344.

van BINSBERGEN, W.M.J., 1992a, Tears of Rain: Ethnicity and history in central western Zambia, Londres/New York: Kegan Paul International.

van BiNSBERGEN, W.M.J., 1992b, 'Making sense of urban space in Francistown, Botswana', contribution au symposium 'Urban Symbolism', Centrum voor Niet-Westerse Studies, Leiden, janvier 1992; sous presse in P. Nas, réd., Urban Images, Leiden, Brill.

van BINSBERGEN, W.M.J., \& M.R. DOORNBOS, réd., 1987, Afrika in spiegelbeeld, Haarlem: In de Knipscheer.

van Binsbergen, W.M.J., \& P.L. Geschiere, 1985a, réd., Old Modes of Production and Capitalist Encroachment, Londres/Boston: Kegan Paul International.

van BINSBERGEN, W.M.J., \& P.L. GESCHIERE 1985b, réd., 'Marxist theory and anthropological practice: The application of French Marxist anthropology in fieldwork', in Binsbergen \& Geschiere 1985a: 235-289.

van BORSELEN, D., 1985, 'Etnisering en etnische belangenbehartiging: Een reactie', Migrantenstudies, 1, 3: 214-224.

van de WERK, J.K., 'Nawoord', in Okot p'Bitek 1980: 257-269.

van der BURG, C, \& P. van der VEER, 1986, 'Pandits, power and profit: Religious organization and the construction of identity among Surinamese Hindus', Ethnic and Racial Studies, 9, 4: 514-528.

van WeTERING, W., 1991, 'A statue for Mother Earth: The politics of multiculturalism in Bijlmermeer, Amsterdam', Contribution à l'atelier 'Etniciteit in Afrika', décembre 1991, Leiden, Afrika-Studiecentrum.

WILMSEN, E.N., 1989, Land filled with flies: A political economy of the Kalahari, Chicago \& Londres: University of Chicago Press. 